\title{
The Effect on the Hip Muscle Activation of the Fall Direction and Knee Position During a Fall
}

\author{
Kwang Jun Lee, PT, Kitaek Lim, PT, Woochol Joseph Choi, PT, PhD \\ Department of Physical Therapy, Injury Prevention and Biomechanics Laboratory, Yonsei University, Wonju, Korea
}

\author{
Article Info \\ Received November 10, 2020 \\ Revised January 8, 2021 \\ Accepted January 11, 2021

\section{Corresponding Author \\ Woochol Joseph Choi \\ E-mail: wcjchoi@yonsei.ac.kr \\ https://orcid.org/0000-0002-6623-3806}

\section{Key Words}

Fall direction

Falls

Hip fracture

Muscle activation

Spontaneous hip fracture
Background: A hip fracture may occur spontaneously prior to the hip impact, due to the muscle pulling force exceeding the strength of the femur.

Objects: We conducted falling experiments with humans to measure the activity of the hip muscles, and to examine how this was affected by the fall type.

Methods: Eighteen individuals fell and landed sideways on a mat, by mimicking video-captured real-life older adults' falls. Falling trials were acquired with three fall directions: forward, backward, or sideways, and with three knee positions at the time of hip impact, where the landing side knee was free of constraint, or contacted the mat or the contralateral knee. During falls, the activities of the iliopsoas (Ilio), gluteus medius (Gmed), gluteus maximus (Gmax) and adductor longus (ADDL) muscles were recorded. Outcome variables included the time to onset, activity at the time of hip impact, and timing of the peak activity with respect to the time of hip impact.

Results: For llio, Gmed, Gmax, and ADDL, respectively, EMG onset averaged 292, 304, 350, and 248 ms after fall initiation. Timing of the peak activity averaged 106,96, 84, and 180 ms prior to the hip impact, and activity at the time of hip impact averaged $72.3,45.2,64.3$, and $63.4 \%$ of the peak activity. Furthermore, the outcome variables were associated with fall direction and/or knee position in all but the iliopsoas muscle.

Conclusion: Our results provide insights on the hip muscle activation during a fall, which may help to understand the potential injury mechanism of the spontaneous hip fracture.

\section{INTRODUCTION}

A hip fracture, which often causes death and permanent disabilities, is a debilitating injury in older adults, and over $90 \%$ of cases are related to a fall [1]. Risk of hip fracture during a fall depends on the bone strength as well as the applied force [2], and a hip fracture occurs when the applied force exceeds the femoral strength at impact. This trauma-based understanding of hip fracture mechanism had informed the development and enhancement of strategies for hip fracture prevention in older adults, and currently available interventions have aimed to reduce the hip impact force during a fall (i.e., hip protector, compliant flooring, safe landing strategies) [3-8]. However, the applied force to the femur during a fall includes muscles' pulling force, which could be large enough to cause a fracture prior to the time of hip impact during a fall. This is a possible scenario due to the facts that (a) physiologically obtainable pulling force of the gluteus medius muscle is estimated at 2-4 times of body weight during activities of daily living [9,10], which remains within the range of femoral strength of older women (between 1,997 and 3,053 N) [11,12], and (b) the hip muscle activity becomes more active during balance recovery when lost balance [13]. This response activity of muscles spanning the hip joint during a fall, which never been explored, may generate a pulling force exceeding the fracture strength during descent to cause a fracture "spontaneously".

Research evidence suggests that the spontaneous hip fracture may account for $5 \%-60 \%$ of hip fractures in older adults [14-16]. Alffram [17] has reported fifteen hip fracture cases occurred with no trauma, and Smith [18] has reported 2 cases that did not involve a fall. Sloan and Holloway [19] has examined 54 hip fracture patients admitted to a local hospital, and 
found that 13 cases (24\%) were due to the leg giving way. Furthermore, evidence suggests that a fracture is common during electroconvulsive therapy with no muscle relaxant, indicating that sudden muscle contraction causes a fracture [20-22]. Collectively, a potential injury mechanism that may explain the spontaneous hip fracture during a fall concerns forceful contraction of hip muscles prior to impact.

Muscle contraction during a fall may be well monitored through electromyography (EMG). However, no simple method has been, so far, established to predict the muscle force from the EMG data, due to the fact that the force magnitude generated by a single muscle can not be measured in vivo (particularly challenging in dynamic condition, where muscle length changes over time) and EMG data often reflect the activity of several other muscles. Despite the limitation, relative comparison is possible (i.e., the higher the EMG value, the greater the force is developed) when normalized to an EMG value of maximal voluntary isometric contraction (MVIC) or a reference EMG value (i.e., peak or average EMG value during an event) [23-25]. Furthermore, the maximum isometric force of a muscle can be estimated as the product of the physiological cross-sectional area (PCSA) and the muscle tension [26]. Collectively, one can estimate the muscle pulling force during a fall, assuming the peak EMG activity of a muscle during a fall is equal to a value recorded when the muscle contracts isometrically and maximally.

Video analyses of real-life falls captured through surveillance cameras in long term care facilities had revealed fall characteristics of older adults, where older adults' falls are a lot different than our traditional notions on falls. For example, a trip or slip was believed to be the number one cause of a fall, but incorrect weight shifting was the most common cause in older adults' falls $[27,28]$. Sideways falls were believed to be the most common (thus most lab-based fall simulations involved sideways perturbations), but the initial fall direction was fairly evenly distributed among forward, backward, sideways, and straight down. Furthermore, the initial fall direction did not necessary reflect landing configuration, and 36\% of falls involved body rotation during a fall (i.e., fall initiated forward but landed sideways) [29,30]. In addition, three common knee positions were observed at the time of hip impact, and the landing side knee was being contacted the ground (43\%), the contralateral knee (23\%), or free of constraint (33\%) [31]. These characteristics should be considered when simulate falls in the laboratory.

Against this background, we conducted falling experiments with humans to measure the activity of hip muscles during a fall. We then examined how the activity was affected by the fall direction and the knee position at the time of hip impact.

\section{MATERIALS AND METHODS}

\section{Subjects}

Eighteen healthy individuals ( 9 males and 9 females) aged between 19 and 36 were participated. Demographics of participants were provided in Table 1. Exclusion criteria included a history of fracture fixation surgery, musculoskeletal conditions (i.e., sprain, strain), and pains or discomforts during a fall. The study protocol was approved by the Institutional Review Board at Yonsei University Mirae Campus, and all subjects agreed to participate by providing a written informed consent form.

\section{Experimental Protocol and Equipment}

Participants self-initiated a fall and landed sideways on a 30 cm thick gymnasium mattress, mimicking typical older adults' falls presented by real-life fall videos or demonstrations provided by investigators [27,31] (Figure 1). Falling trials were acquired with three initial fall directions: forward, backward and sideways. Trials were also acquired with three knee positions at the time of hip impact, where the landing side knee was being contacted the mat (KOM), the contralateral knee (KOK), or free of constraint (KF). Participants were instructed to "fall as natural as possible" when a verbal cue was provided by the investigator, and no other instructions were given. When they became comfortable in mimicking the falls on the mat, electrodes of surface EMG (TeleMyo DTS; Noraxon Inc., Scottsdale, AZ, USA) were placed on the belly of iliopsoas (Ilio), gluteus medius (Gmed), gluteus maximus (Gmax), and adductor longus (ADDL). Reflective markers were placed at the anterior superior iliac spine, sacrum, and posterior superior iliac spine

Table 1. Demographics of participants

\begin{tabular}{lcc}
\hline & Male $(\mathrm{n}=9)$ & Female $(\mathrm{n}=9)$ \\
\hline Age $(\mathrm{y})$ & $24.2 \pm 5.0$ & $24.0 \pm 2.7$ \\
Body weight $(\mathrm{kg})$ & $71.4 \pm 11.3$ & $58.2 \pm 7.4$ \\
Body height $(\mathrm{cm})$ & $171.9 \pm 7.0$ & $161.7 \pm 2.8$ \\
Body mass index $\left(\mathrm{kg} / \mathrm{m}^{2}\right)$ & $24.2 \pm 3.7$ & $22.3 \pm 2.8$ \\
\hline
\end{tabular}

Values are presented as mean \pm standard deviation. 


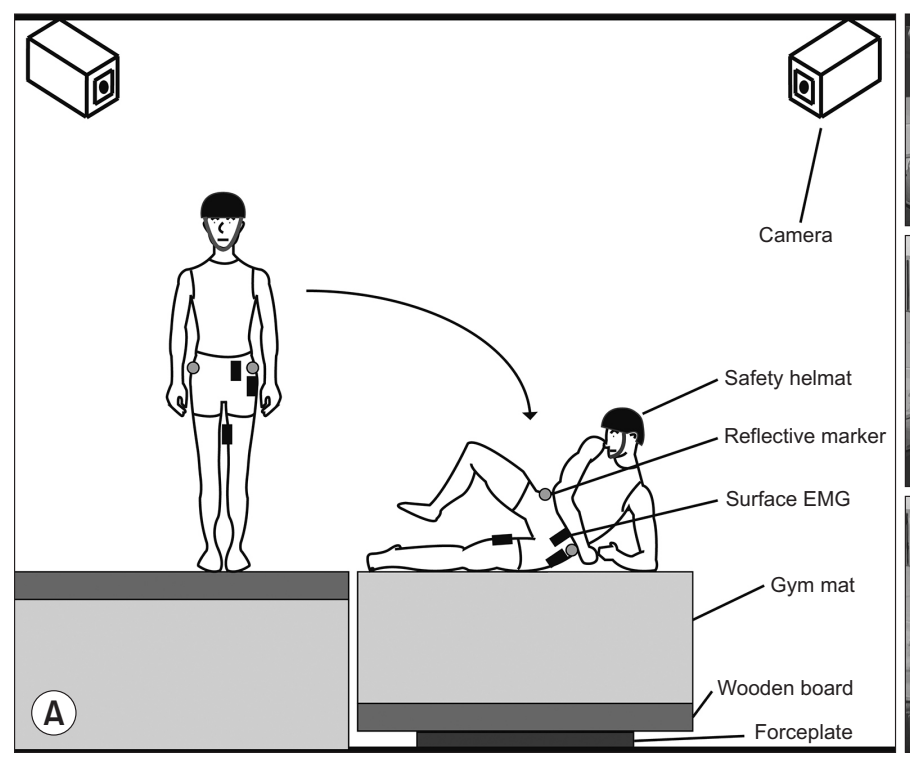

in order to monitor fall kinematics through motion capture system (Vicon Motion Systems, Oxford, UK). A force plate was placed under the mat, and recorded fall kinetics during a fall (model OR6-7-2000; AMTI, Waltham, MA, USA). EMG, motion and force data were synchronized, and collected with a sampling rate of 3,000, 200, and 3,000 Hz, respectively. Three falling trials were acquired for each fall condition, and the order of presentation of condition was randomized.

\section{Data Analysis}

Outcome variables included the time to onset, activity at the time of hip impact, and timing of the peak activity with respect to the time of hip impact. Raw EMG data were low-pass filtered with a cutoff frequency of $1,000 \mathrm{~Hz}$, full-wave rectified, and smoothed using a root mean square with a moving window of 100 ms. The processed, time-series EMG data were, then, normalized by dividing by the maximum EMG value occurring between "fall initiation" and "hip impact" [32]. The instants of fall initiation and hip impact were defined as the moment when a pelvis marker started to move downward and traveled $5 \%$ of body height (i.e., $8.5 \mathrm{~cm}$ for an individual of height 170 $\mathrm{cm}$ ), and as the time when the peak vertical force occurred, respectively (Figure 2 ). The time to onset was defined as the time when EMG activity exceeded 3 standard deviations above its average value in the preceding $300 \mathrm{~ms}$. All analyses were conducted with customized Matlab routines (Matlab R2019b; MathWorks Inc., Natick, MA, USA).

For statistical analyses, one-way ANOVA was used to test if

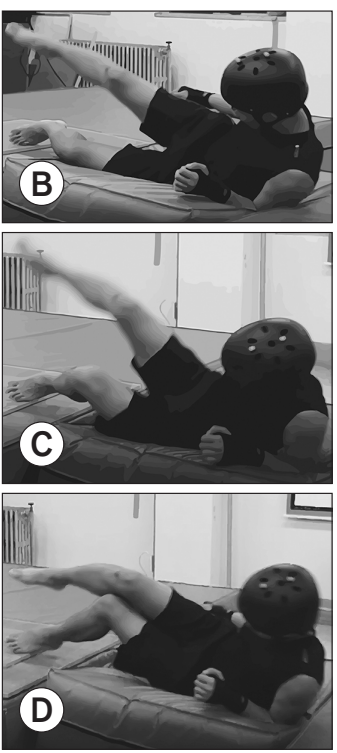

Figure 1. Experimental setup. (A) Participants self-initiated falls and landed sideways on a $30 \mathrm{~cm}$ thick mattress. The falling trials involved one of three knee positions at the time of hip impact: (B) knee on the mat, (C) knee free of constraint, and (D) knee contacts the contralateral knee. The activity of hip muscles, fall kinematics and kinetics were measured through surface electromyography, motion capture system and force plate, respectively, during a fall. $E M G$, electromyography.

these outcome variables were associated with the hip muscle (4 levels). Two-way ANOVA was also used to examine whether the outcome variables were associated with the fall direction (3 levels) and the knee position at the time of hip impact (3 levels). When main effects existed, pair-wise comparison was conducted using a Bonferroni correction. All analyses were conducted with a significance level of alpha $=0.05$ using SPSS 26 (IBM corp., Armonk, NY, USA).

\section{RESULTS}

\section{One-way ANOVA}

The EMG onset was associated with the hip muscle $(F=5.28$, $\mathrm{p}=0.003)$. The average onset was smallest in ADDL (248 \pm 73 $\mathrm{ms}$ after fall initiation), followed by Ilio (292 $\pm 116 \mathrm{~ms})$, Gmed (304 $\pm 96 \mathrm{~ms})$ and $\mathrm{Gmax}(350 \pm 116 \mathrm{~ms})$ (Table 2, Figure 3).

The timing of the peak activity was associated with the hip muscle $(F=6.51, p=0.005)$. For Ilio, Gmed, Gmax, and ADDL, respectively, the peak activity occurred $106 \pm 74,96 \pm 21,84$ \pm 51 , and $180 \pm 120 \mathrm{~ms}$ prior to the hip impact.

The activity at the time of hip impact was associated with the hip muscle $(\mathrm{F}=15.3, \mathrm{p}=0.0005)$. The average activity was greatest in Ilio $(72.3 \pm 8.1 \%$ of the peak activity), followed by $\operatorname{Gmax}(64.3 \pm 11.7 \%), \operatorname{ADDL}(63.4 \pm 14.2 \%)$, and Gmed (45.2 $\pm 12.5 \%)$.

\section{Two-way ANOVA}

For Ilio, none of our outcome variables were associated with 


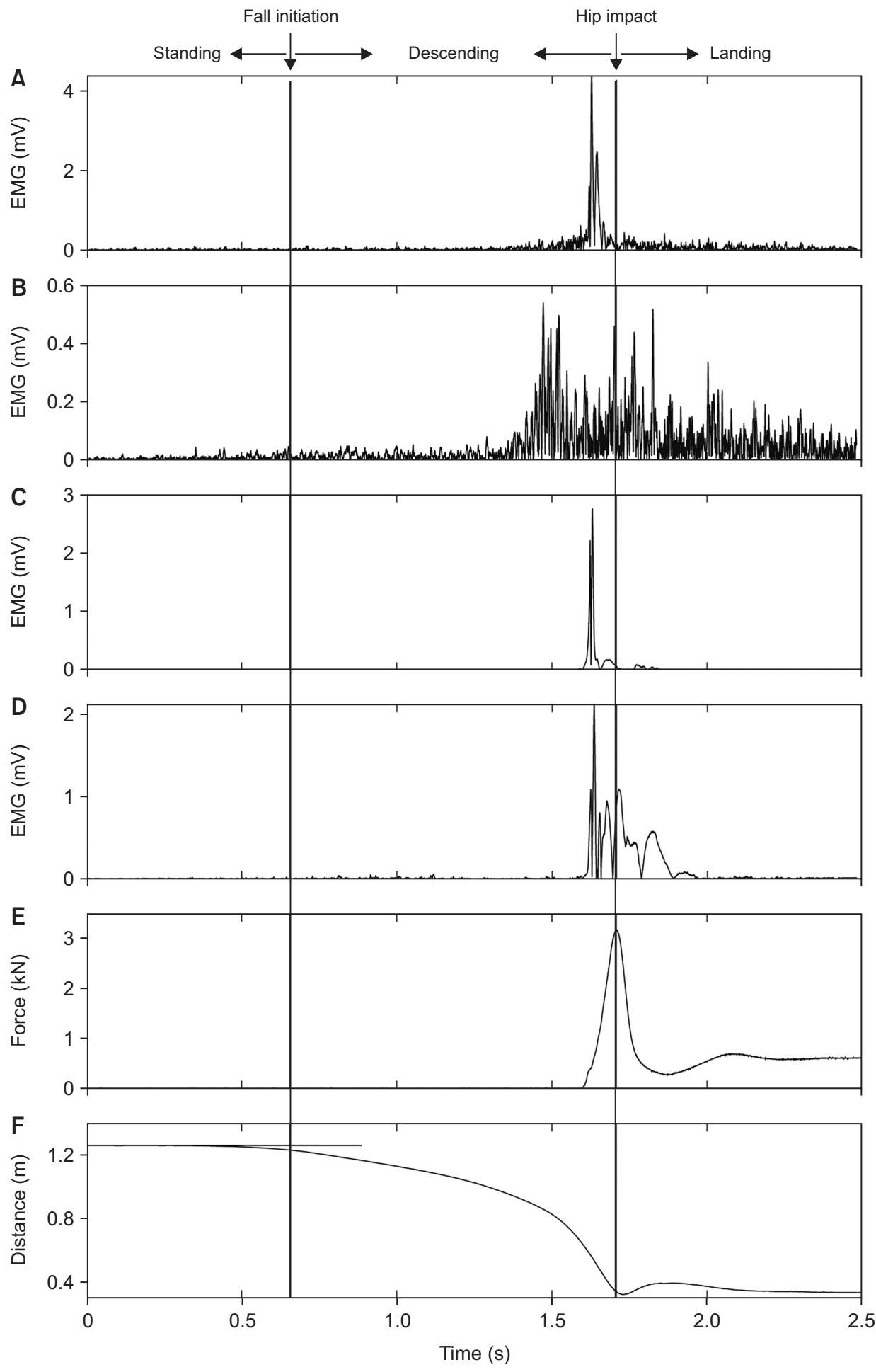

Figure 2. Raw sample data from a particular participant when fell forward and landed sideways with the landing side knee free of constraint at the time of hip impact. All four muscles -(A) iliopsoas, (B) adductor longus, (C) gluteus medius, and (D) gluteus maximus- reached their maximum contraction prior to the hip impact. Changes in vertical impact force (E) and vertical position of a pelvis marker (F) were used to define the moments of fall initiation and hip impact, respectively (see details in texts). EMG, electromyography. the fall direction $(p>0.085)$ and the knee position $(p>0.375)$ (Table 2).

For Gmed, the activity at the time of hip impact was associated with the fall direction $(\mathrm{F}=8.8, \mathrm{p}=0.001)$ and the knee position $(\mathrm{F}=6.1, \mathrm{p}=0.005)$. On average, the activity was $27.1 \%$ greater in backward than forward falls, and $42.7 \%$ greater in KOM than KF (52.3 vs. $41.1 \%$; 52.8 vs. $36.9 \%$, respectively).
For Gmax, the EMG onset was associated with the fall direction $(\mathrm{F}=3.3, \mathrm{p}=0.046)$, but not with the knee position $(\mathrm{F}=$ $1.1, p=0.317)$. The timing of the peak activity was associated with the knee position $(\mathrm{F}=7.3, \mathrm{p}=0.002)$, but not with the fall direction $(\mathrm{F}=1.0, \mathrm{p}=0.334)$. The activity at the time of hip impact was associated with the knee position $(\mathrm{F}=13.2, \mathrm{p}=$ 0.00005), but not with the fall direction $(\mathrm{F}=1.8, \mathrm{p}=0.175)$. 
Table 2. Average values of outcome variables

\begin{tabular}{|c|c|c|c|c|c|c|c|c|c|c|}
\hline \multirow{2}{*}{\multicolumn{2}{|c|}{ Outcome variables }} & \multicolumn{3}{|c|}{ Forward } & \multicolumn{3}{|c|}{ Sideways } & \multicolumn{3}{|c|}{ Backward } \\
\hline & & $\mathrm{KF}$ & KOK & KOM & KF & KOK & KOM & $\mathrm{KF}$ & KOK & KOM \\
\hline \multirow[t]{4}{*}{ Onset time (ms) } & Ilio & $324 \pm 160$ & $313 \pm 212$ & $245 \pm 105$ & $289 \pm 153$ & $338 \pm 259$ & $262 \pm 214$ & $266 \pm 182$ & $282 \pm 172$ & $305 \pm 197$ \\
\hline & Gmed & $276 \pm 114$ & $304 \pm 120$ & $268 \pm 116$ & $236 \pm 91$ & $333 \pm 119$ & $337 \pm 305$ & $304 \pm 207$ & $369 \pm 246$ & $307 \pm 177$ \\
\hline & Gmax & $344 \pm 202$ & $415 \pm 207$ & $330 \pm 225$ & $296 \pm 145$ & $302 \pm 149$ & $291 \pm 168$ & $415 \pm 285$ & $417 \pm 326$ & $338 \pm 125$ \\
\hline & ADDL & $346 \pm 242$ & $289 \pm 153$ & $192 \pm 75$ & $221 \pm 85$ & $234 \pm 128$ & $205 \pm 76$ & $259 \pm 150$ & $254 \pm 94$ & $229 \pm 143$ \\
\hline \multirow{4}{*}{$\begin{array}{l}\text { Activity at the time of } \\
\text { hip impact (\%) }\end{array}$} & Ilio & $70.4 \pm 16.6$ & $72.3 \pm 17.3$ & $69.7 \pm 17.7$ & $74.6 \pm 17.5$ & $79.1 \pm 11.1$ & $67.5 \pm 17.6$ & $72.2 \pm 23.5$ & $70.2 \pm 24.6$ & $74.5 \pm 12.4$ \\
\hline & Gmed & $33.8 \pm 15.8$ & $41.1 \pm 21.6$ & $48.4 \pm 19.7$ & $31.5 \pm 15.9$ & $42.6 \pm 23.7$ & $53.0 \pm 21.4$ & $45.6 \pm 21.2$ & $54.4 \pm 26.8$ & $56.8 \pm 15.4$ \\
\hline & Gmax & $54.8 \pm 22.1$ & $51.0 \pm 22.0$ & $74.0 \pm 17.4$ & $56.5 \pm 20.4$ & $64.4 \pm 19.1$ & $77.0 \pm 18.5$ & $64.7 \pm 22.7$ & $58.3 \pm 25.4$ & $77.7 \pm 19.7$ \\
\hline & ADDL & $62.6 \pm 21.6$ & $68.4 \pm 20.3$ & $60.8 \pm 24.2$ & $62.6 \pm 19.5$ & $64.2 \pm 23.4$ & $56.8 \pm 19.4$ & $64.1 \pm 19.9$ & $66.5 \pm 27.7$ & $64.8 \pm 21.6$ \\
\hline \multirow{4}{*}{$\begin{array}{l}\text { Timing of the peak } \\
\text { activity with respect } \\
\text { to the hip impact } \\
\text { (ms) }\end{array}$} & Ilio & $81 \pm 45$ & $100 \pm 88$ & $85 \pm 67$ & $80 \pm 54$ & $105 \pm 163$ & $96 \pm 95$ & $165 \pm 288$ & $163 \pm 226$ & $82 \pm 59$ \\
\hline & Gmed & $105 \pm 22$ & $93 \pm 26$ & $80 \pm 26$ & $107 \pm 29$ & $98 \pm 41$ & $98 \pm 74$ & $101 \pm 69$ & $99 \pm 69$ & $90 \pm 58$ \\
\hline & Gmax & $75 \pm 24$ & $95 \pm 76$ & $51 \pm 26$ & $115 \pm 105$ & $75 \pm 38$ & $62 \pm 61$ & $87 \pm 72$ & $143 \pm 177$ & $57 \pm 73$ \\
\hline & ADDL & $160 \pm 114$ & $169 \pm 116$ & $143 \pm 108$ & $255 \pm 384$ & $192 \pm 170$ & $165 \pm 93$ & $171 \pm 153$ & $158 \pm 132$ & $214 \pm 297$ \\
\hline
\end{tabular}

Values are presented as mean \pm standard deviation. KF, knee free of constraint; KOK, knee contacted the contralateral knee; KOM, knee contacted the mat; Ilio, iliopsoas muscle; Gmed, gluteus medius muscle; Gmax, gluteus maximus muscle; ADDL, adductor longus muscle.

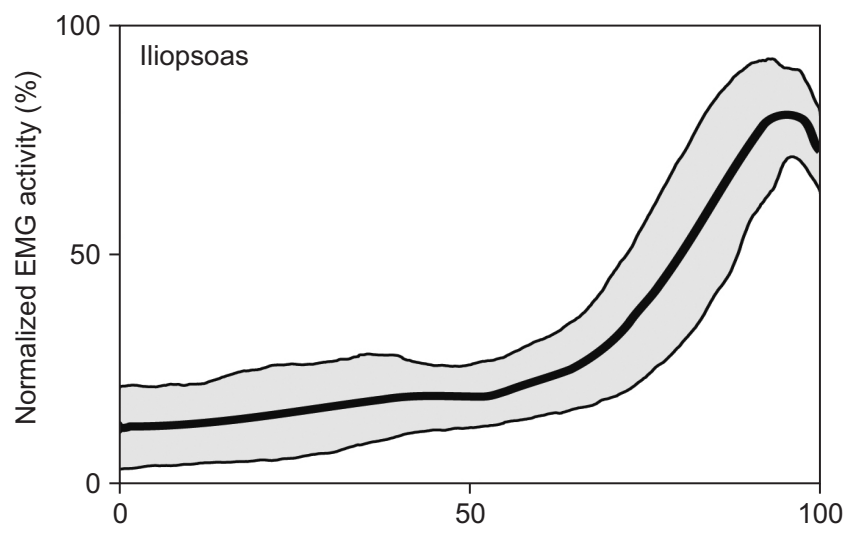

Fall time (\%)

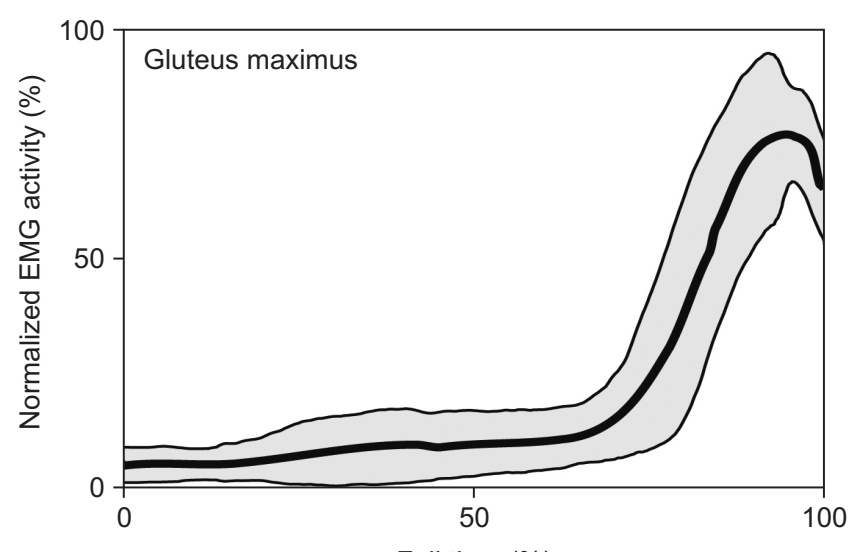

Fall time (\%)

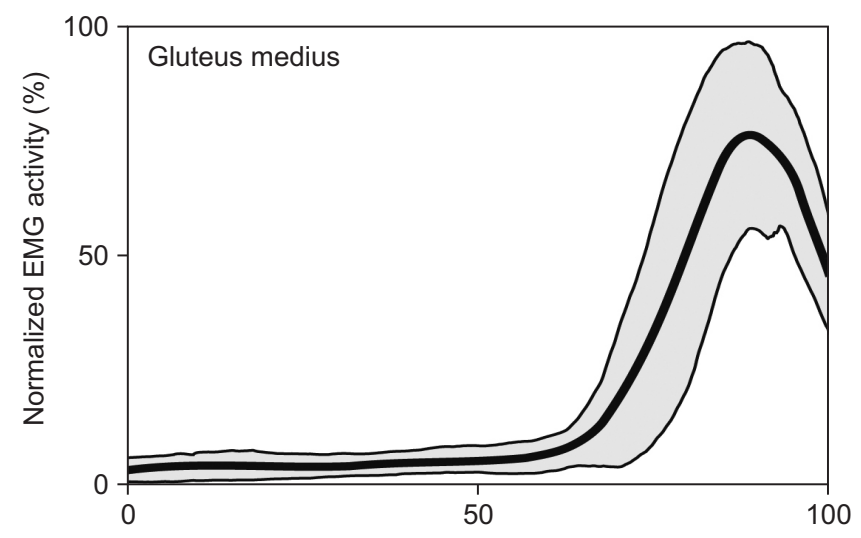

Fall time (\%)

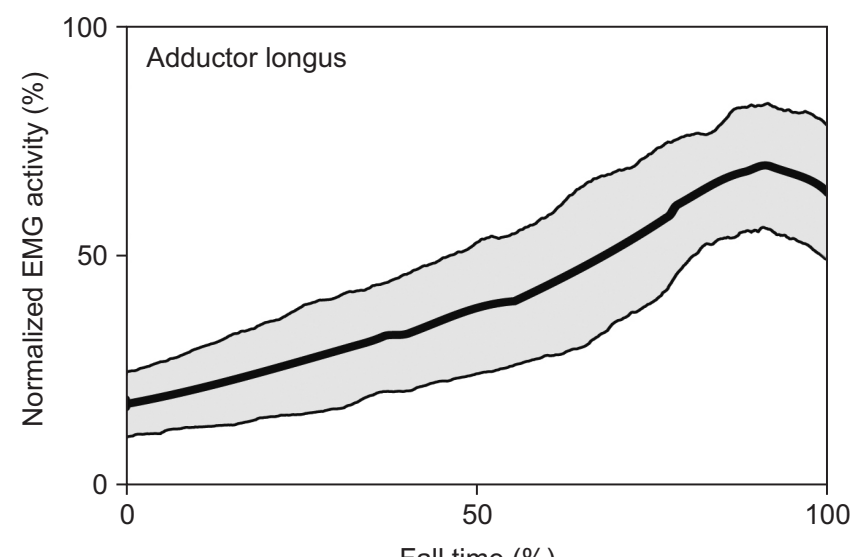

Fall time (\%)

Figure 3. Average EMG activity (thick line) across all 486 falling trials with +/- 1 standard deviation (thin line). A EMG sampling rate was 3,000 Hz and average fall time between fall initiation and hip impact was 979 ms (about 1 second). To superimpose and average, the EMG data were resampled with a rate of $3,000 \mathrm{~Hz}$ using a Matlab function "resample". EMG, electromyography.

For ADDL, the EMG onset was associated with the fall direction $(\mathrm{F}=3.2, \mathrm{p}=0.05)$ and the knee position $(\mathrm{F}=4.2, \mathrm{p}=$ 0.023). On average, the onset was $24.8 \%$ earlier in sideways than forward falls, and 32\% earlier in KOM than KF (221 vs. 276 ms; 209 vs. 276 ms, respectively). 


\section{DISCUSSION}

The purpose of this study was to describe the activation pattern of hip muscles during a fall. We found that, given a time available during a fall (979 ms, average time between fall initiation and hip impact across all trials), hip muscles started to contract, on average, $299 \mathrm{~ms}$ after fall initiation, and reached the maximum contraction at $117 \mathrm{~ms}$ prior to the hip impact. Furthermore, the hip muscles contracted $61.3 \%$ of their maximum at the time of hip impact (Figure 3). These baseline measures should be informative for the development of EMG-based fall-injury prevention strategies (i.e., pre-impact fall detection algorithms).

To place the results in context of an injury mechanism of the spontaneous hip fracture in frail population, we need to introduce three assumptions. First, the response activities of hip muscles during a fall reaches their maximum during a fall. Second, the measured peak EMG activity during a fall represents the maximum isometric contraction of a muscle at a certain hip joint angle. Third, the maximum isometric force values of hip muscles available in the literature [26] are equal to those of participants in our study. Under this scenario, the maximum isometric force of Ilio, Gmed, Gmax, and ADDL refer to $848.8,2,199.6,1,852.6$, and 1,296.9 N, respectively, which close to the average fracture strength of the proximal femur in older women $(2,826 \pm 424 \mathrm{~N})[11,12,33-36]$. Furthermore, the maximum force occurs, on average, at $117 \mathrm{~ms}$ prior to the hip impact, and $61.3 \%$ of the maximum force applies to the proximal femur at the time of hip impact. Similar efforts have been made to understand the potential harmful effect of the muscle pulling force prior to the hip impact during a fall. Choi et al. [31] have used a mechanical hip impact simulator, and found that the hip abductor muscle force generates compressive and tensile stresses at the femoral neck prior to the hip impact $\left(0.1-4.6 \mathrm{~N} / \mathrm{m}^{2}\right)$, which remains within the range of the ultimate stresses of the trabecular bone of the proximal femur (0.4-10.0 $\mathrm{N} / \mathrm{m}^{2}$ ) [37-42]. Collectively, the results suggest the high likelihood of spontaneous hip fracture during a fall, particularly in frail older population.

Another purpose of this study was to examine whether the muscle activation pattern differed among four hip muscles, and how the muscle activity was affected by the type of a fall. Our two-way ANOVA suggested that the muscle activation pattern depended on how they fall and land in all but the iliopsoas muscle, indicating that the hip flexor muscles non-significantly contribute to joint kinematics (i.e., hip flexion/extension/rotation) during fall conditions that we have examined. Furthermore, our one-way ANOVA suggested that the iliopsoas muscle reached the maximum contraction $106 \mathrm{~ms}$ prior to the hip impact, and the activity at the time of hip impact was greatest during fall conditions that we have tested. Collectively, the iliopsoas muscle could be the one which creates the greatest load on the femur, regardless of how they fall and land. This may support a spontaneous hip fracture mechanism, where the femoral neck fractures due to the torsional stress caused by the forceful contraction of the iliopsoas muscle during a fall [43].

Our results should be interpreted in light of several limitations. First, while older adults' falls were replicated in the laboratory, falls were self-initiated on a mat. Therefore, the hip muscle activity might be underestimated. The falls may be induced artificially (i.e., perturbation platform), but the perturbation involving the high acceleration and velocity (often necessary to cause a fall) may result in overestimation. Second, our simulation of falls did not address the cause of a fall (i.e., slip, trip, faint) and the activity at the time of a fall (i.e., walking, tuning, rising from a chair), which may affect the hip muscle activation pattern. Third, we did not control upper extremities during a fall, which may affect lower extremity muscle activations. While the hand, wrist and elbow positions and impact timing were consistent across all trials, the potential effects should be considered when interpret the results. Finally, we only included healthy young individuals due to safety reasons. Therefore, experiments with older adults, involving a safer experimental design (i.e., near fall-initiate a fall but catch them to avoid impact) under a variety of fall circumstances, are warranted.

\section{CONCLUSIONS}

In summary, our results provide insights on the hip muscle activation pattern during a fall, which may help to understand the potential injury mechanism of the spontaneous hip fracture in older adults.

\section{ACKNOWLEDGEMENTS}

This work was supported by the "Brain Korea 21 FOUR Project”, the Korean Research Foundation for Department of Phys- 
ical Therapy in the Graduate School of Yonsei University, and by Yonsei University Mirae Campus Future-Leading Research Initiative (\#2019-62-0023).

\section{CONFLICTS OF INTEREST}

No potential conflict of interest relevant to this article was reported.

\section{AUTHOR CONTRIBUTIONS}

Conceptualization: WJC. Data curation: KJL, KL, WJC. Formal analysis: KJL, KL, WJC. Funding acquisition: WJC. Investigation: KJL, KL, WJC. Methodology: KJL, KL, WJC. Project administration: KJL, KL, WJC. Resources: KJL, KL, WJC. Software: KJL, KL, WJC. Supervision: WJC. Validation: KJL, KL, WJC. Visualization: WJC. Writing - original draft: KJL, WJC. Writing - review \& editing: KJL, KL, WJC.

\section{ORCID}

Kwang Jun Lee, https://orcid.org/0000-0001-5497-903X

Kitaek Lim, https://orcid.org/0000-0001-5475-3196

\section{REFERENCES}

1. Grisso JA, Schwarz DF, Wishner AR, Weene B, Holmes JH, Sutton RL. Injuries in an elderly inner-city population. J Am Geriatr Soc 1990;38(12):1326-31.

2. Bouxsein ML, Szulc P, Munoz F, Thrall E, Sornay-Rendu E, Delmas PD. Contribution of trochanteric soft tissues to fall force estimates, the factor of risk, and prediction of hip fracture risk. J Bone Miner Res 2007;22(6):825-31.

3. Choi WJ, Hoffer JA, Robinovitch SN. The effect of positioning on the biomechanical performance of soft shell hip protectors. J Biomech 2010;43(5):818-25.

4. Choi WJ, Hoffer JA, Robinovitch SN. Effect of hip protectors, falling angle and body mass index on pressure distribution over the hip during simulated falls. Clin Biomech (Bristol, Avon) 2010;25(1):63-9.

5. Lim KT, Choi WJ. Effect of fall characteristics on the severity of hip impact during a fall on the ground from standing height. Osteoporos Int 2020;31 (9):1713-9.

6. Laing AC, Robinovitch SN. The force attenuation provided by hip protectors depends on impact velocity, pelvic size, and soft tissue stiffness. J Biomech Eng 2008;130(6):061005.

7. Laing AC, Robinovitch SN. Low stiffness floors can attenuate fall-related femoral impact forces by up to $50 \%$ without substantially impairing balance in older women. Accid Anal Prev 2009;41(3):642-50.

8. Robinovitch SN, Evans SL, Minns J, Laing AC, Kannus P, Cripton PA, et al. Hip protectors: recommendations for biomechanical testing--an international consensus statement (part I). Osteoporos Int 2009;20(12):1977-88.

9. Frankel VH, Burstein AH. Orthopaedic biomechanics: the application engineering to the musculoskeletal system. Philadelphia: Lea \& Febiger; 1970.

10. Patriarco AG, Mann RW, Simon SR, Mansour JM. An evaluation of the approaches of optimization models in the prediction of muscle forces during human gait. J Biomech 1981;14(8):51325.

11. Bouxsein ML, Coan BS, Lee SC. Prediction of the strength of the elderly proximal femur by bone mineral density and quantitative ultrasound measurements of the heel and tibia. Bone 1999;25(1):49-54.

12. Pulkkinen $P$, Jämsä T, Lochmüller EM, Kuhn V, Nieminen MT, Eckstein F. Experimental hip fracture load can be predicted from plain radiography by combined analysis of trabecular bone structure and bone geometry. Osteoporos Int 2008;19(4):547-58.

13. Horak FB, Nashner LM. Central programming of postural movements: adaptation to altered support-surface configurations. J Neurophysiol 1986;55(6):1369-81.

14. Parker MJ, Twemlow TR. Spontaneous hip fractures, $44 / 872$ in a prospective study. Acta Orthop Scand 1997;68(4):325-6.

15. Muckle DS. Iatrogenic factors in femoral neck fractures. Injury 1976;8(2):98-101.

16. Parkkari J, Kannus P, Palvanen M, Natri A, Vainio J, Aho H, et al. Majority of hip fractures occur as a result of a fall and impact on the greater trochanter of the femur: a prospective controlled hip fracture study with 206 consecutive patients. Calcif Tissue Int 1999;65(3):183-7.

17. Alffram PA. An epidemiologic study of cervical and trochanteric fractures of the femur in an urban population. Analysis of 1,664 cases with special reference to etiologic factors. Acta Orthop Scand Suppl 1964; 35 Suppl 65:1-109.

18. Smith LD. Hip fractures; the role of muscle contraction or intrinsic forces in the causation of fractures of the femoral 
neck. J Bone Joint Surg Am 1953;35-A(2):367-83.

19. Sloan J, Holloway G. Fractured neck of the femur: the cause of the fall? Injury 1981;13(3):230-2.

20. Kelly JP. Fractures complicating electro-convulsive therapy and chronic epilepsy. J Bone Joint Surg Br 1954;36-B(1):709.

21. Nott MR, Watts JS. A fractured hip during electro-convulsive therapy. Eur J Anaesthesiol 1999;16(4):265-7.

22. Newbury CL, Etter LE. Clarification of the problem of vertebral fractures from convulsive therapy. I. Incidence. AMA Arch Neurol Psychiatry 1955;74(5):472-8.

23. De Luca CJ. The use of surface electromyography in biomechanics. J Appl Biomech 1997;13(2):135-63.

24. Inman VT, Ralston HJ, Saunders JB, Feinstein B, Wright EW Jr. Relation of human electromyogram to muscular tension. Electroencephalogr Clin Neurophysiol 1952;4(2):187-94.

25. Bigland B, Lippold OC. The relation between force, velocity and integrated electrical activity in human muscles. J Physiol 1954;123(1):214-24.

26. Arnold EM, Ward SR, Lieber RL, Delp SL. A model of the lower limb for analysis of human movement. Ann Biomed Eng 2010;38(2):269-79.

27. Robinovitch SN, Feldman F, Yang Y, Schonnop R, Leung PM, Sarraf T, et al. Video capture of the circumstances of falls in elderly people residing in long-term care: an observational study. Lancet 2013;381(9860):47-54.

28. Yang Y, Schonnop R, Feldman F, Robinovitch SN. Development and validation of a questionnaire for analyzing real-life falls in long-term care captured on video. BMC Geriatr 2013;13:40.

29. Schonnop R, Yang Y, Feldman F, Robinson E, Loughin M, Robinovitch SN. Prevalence of and factors associated with head impact during falls in older adults in long-term care. CMAJ 2013;185(17):E803-10.

30. Yang Y, Mackey DC, Liu-Ambrose T, Feldman F, Robinovitch SN. Risk factors for hip impact during real-life falls captured on video in long-term care. Osteoporos Int 2016;27(2):53747.

31. Choi WJ, Cripton PA, Robinovitch SN. Effects of hip abductor muscle forces and knee boundary conditions on femoral neck stresses during simulated falls. Osteoporos Int 2015;26(1):291-301.
32. Yang JF, Winter DA. Electromyographic amplitude normalization methods: improving their sensitivity as diagnostic tools in gait analysis. Arch Phys Med Rehabil 1984;65(9):517-21.

33. Cheng XG, Lowet G, Boonen S, Nicholson PH, Brys P, Nijs J, et al. Assessment of the strength of proximal femur in vitro: relationship to femoral bone mineral density and femoral geometry. Bone 1997;20(3):213-8.

34. Cheng XG, Lowet G, Boonen S, Nicholson PH, Van der Perre G, Dequeker J. Prediction of vertebral and femoral strength in vitro by bone mineral density measured at different skeletal sites. J Bone Miner Res 1998;13(9):1439-43.

35. Lochmüller EM, Groll O, Kuhn V, Eckstein F. Mechanical strength of the proximal femur as predicted from geometric and densitometric bone properties at the lower limb versus the distal radius. Bone 2002;30(1):207-16.

36. Pulkkinen P, Eckstein F, Lochmüller EM, Kuhn V, Jämsä T. Association of geometric factors and failure load level with the distribution of cervical vs. trochanteric hip fractures. J Bone Miner Res 2006;21(6):895-901.

37. Fyhrie DP, Schaffler MB. Failure mechanisms in human vertebral cancellous bone. Bone 1994;15(1):105-9.

38. Kopperdahl DL, Keaveny TM. Yield strain behavior of trabecular bone. J Biomech 1998;31(7):601-8.

39. Carter DR, Hayes WC. The compressive behavior of bone as a two-phase porous structure. J Bone Joint Surg Am 1977;59(7):954-62.

40. Galante J, Rostoker W, Ray RD. Physical properties of trabecular bone. Calcif Tissue Res 1970;5(3):236-46.

41. Weaver JK, Chalmers J. Cancellous bone: its strength and changes with aging and an evaluation of some methods for measuring its mineral content. J Bone Joint Surg Am 1966;48(2):289-98.

42. Ebbesen EN, Thomsen JS, Beck-Nielsen H, Nepper-Rasmussen HJ, Mosekilde L. Age- and gender-related differences in vertebral bone mass, density, and strength. J Bone Miner Res 1999;14(8):1394-403.

43. Yang KH, Shen KL, Demetropoulos CK, King AI, Kolodziej P, Levine RS, et al. The relationship between loading conditions and fracture patterns of the proximal femur. J Biomech Eng 1996;118(4):575-8. 